\title{
Romberg Integration: A Symbolic Approach with Mathematica
}

\author{
Ali Yazıcı ${ }^{1}$, Tanıl Ergenç ${ }^{2}$, and Irfan Altas ${ }^{3}$ \\ 1 Computer Engineering Department, Atilim University, Ankara - Turkey \\ aliyazici@atilim.edu.tr \\ 2 Mathematics Department, Middle East Technical University, Ankara - Turkey \\ tanil@metu.edu.tr \\ 3 School of Information Studies, Wagga Wagga - Australia \\ ialtas@csu.edu.au
}

\begin{abstract}
Higher order approximations of an integral can be obtained from lower order ones in a systematic way. For 1-D integrals Romberg Integration is an example which is based upon the composite trapezoidal rule and the well-known Euler-Maclaurin expansion of the error. In this work, Mathematica is utilized to illustrate the method and the underlying theory in a symbolic fashion. This approach seems plausible for discussing integration in a numerical computing laboratory environment.
\end{abstract}

\section{Introduction}

In the last decade, there has been a great interest in using technology in education. The authors of this paper have been involved in teaching with some symbolic algebra packages such as Mathematica and Maple. Use of a symbolic package in teaching mathematics has had a positive impact on student learning. A student survey in a calculus course revealed that majority of students made use of a symbolic package to verify their symbolic and numerical work, simplify certain mathematical derivations, and, to some extent, set assistance in proving simple theorems.

Here, an elegant numerical method, the so-called Romberg integration is considered to demonstrate the use and power of symbolic packages to convey various ideas and concepts. Authors' teaching experience has revealed that students feel more comfortable with the underlying theory if the discussion is supported with a symbolic code. This approach helps students in great extent to follow and verify the mathematical derivations and theoretical justifications easily in a laboratory environment.

In this work, Romberg integration is taken as a case study. This approach may be applicable to similar numerical and mathematical techniques. Any symbolic package would serve the purposes of this study. However, because of its popularity in symbolic computations, and availability in the authors' environment, Mathematica was chosen.

In Section 2, the Romberg integration is summarized. Then, in Section 3 a Mathematica implementation is provided. Sections 4 and 5 demonstrate the 
details of the method symbolically through a set of appropriate Mathematica commands. A discussion about error is given in Section 6. In Section 7, a dynamic implementation is provided followed by Section 8 where cautious extrapolation for treating singularities is discussed. The final section is devoted to a general discussion and conclusions.

\section{Romberg Integration}

The Romberg method approximates the integral, I, below using the linear combinations of well-known trapezoidal sums $T_{i}^{1}$ 's in order to achieve higher orders.

$$
I=\int_{a}^{b} f(x) d x, \quad a, b \in R, \quad f \in C^{k}[a, b]
$$

The method is based on the Euler-Maclaurin asymptotic error expansion formula and the Richardson extrapolation to the limit [1]. Romberg [2] has been the first to formulate the Richardson's method for automatic calculations.

Each trapezoidal sum (of polynomial order 1) is defined as

$$
T_{i}^{1}=\frac{b-a}{2^{i}}\left[f[a]+f[b]+2 \sum_{j=1}^{2^{i-1}-1} f\left[x_{j}\right]\right]
$$

for $i=1,2, \ldots, n$ (n maximum level of subdivision), $x_{j}=x_{o}+j h, j=1,2, \ldots, i$ and $h=(b-a) / 2^{i-1}$. Note that for the $i$ th subdivision of the interval $x_{o}=a$, and $x_{i}=b$. The computation starts with $T_{1}^{1}$ on the interval $[a, b]$, and $T_{2}^{1}, T_{3}^{1}$, and so on are computed by successively halving the interval and applying the basic rule to each subinterval formed. In this subdivision process the Romberg sequence $\left\{1,2,4,8, \ldots 2^{k}, \ldots\right\}$ is utilized to bisect the interval. Other subdivision sequences are also possible [3]. However, Romberg sequence provides full overlapping of the nodes of integration from one extrapolation level to another. Obviously, as the number of subintervals increases, the accuracy of approximations to I improves.

The triangular Romberg table can be constructed using $T_{i}^{j}$ values as

$$
\begin{aligned}
& T_{1}^{1} \\
& T_{2}^{1} T_{2}^{2} \\
& \vdots \quad \vdots \quad \vdots \quad \vdots \quad \ddots \\
& T_{n}^{1} T_{n}^{2} T_{n}^{3} T_{n}^{4} \cdots T_{n}^{n}
\end{aligned}
$$

where $T_{i}^{j}$ 's are obtained from

$$
T_{i}^{j}=\frac{4^{j-1} T_{i}^{j-1}-T_{i-1}^{j-1}}{4^{j-1}-1}, i=2,3, \cdots, n \text { and } j=2,3, \cdots, i
$$

It is well known that the entries in the second column of (3) are composite Simpson approximations [4]. The third column entries are composite approximations based on the Newton interpolatory formulae. Since the first column 
entries are first order approximations to I, trapezoidal sums are exact whenever the integrand $f(x)$ is linear in $x$. All diagonal sequences converge to I, provided that the 1st column also converges as well [5]. Moreover, if column $\mathrm{k}$ is of order $\mathrm{p}$ then the column $k+1$ entries are of order $p+2$. This could easily be justified using the asymptotic error expansion formula (even in powers of $h$ ):

$$
I-T_{i}^{1}=c_{1} h^{2}+c_{2} h^{4}+\cdots+c_{k} h^{2 k}+O\left(h^{2 k+2}\right), \quad h=(b-a) / 2^{i-1}
$$

The $c_{i}$ 's are constants (i.e. independent of $h$ ). Obviously, as $h$ approaches to zero, $T_{j}^{1}$ converges to $I$. For singular integrals this expansion is not valid and it takes different forms depending on the nature of singularity [6].

The identities in (4) and (5) can be utilized to show that

$$
I-T_{2}^{2}=I-\frac{4 T_{2}^{1}-T_{1}^{1}}{3}=-\frac{1}{4} c_{2} h_{1}^{4}+O\left(h_{1}^{6}\right)
$$

which demonstrates that error is of $O\left[h^{4}\right]$ and, hence, a speed-up in the convergence to I.

In the next section, a Mathematica function is provided to verify the computations above in a symbolic computation environment.

\section{Symbolic Romberg Integration}

Symbolic packages are heavily used in engineering research and as an instructional tool in education (see for example [7, and [8])with an emphasis on computational aspects.

In a teaching environment, a symbolic code could be quite beneficial to illustrate the difficult concepts of a problem. For example, some of the theoretical aspects of the Romberg integration method, such as the justification of the asymptotic error expansion, and the discussion on the convergence may become easy once the symbolic power is facilitated.

Many sophisticated library functions are available for numerical integration (see for example Mathematica's Integrate function). The main objective of this study though, is to illustrate the details of a numerical technique by utilizing the symbolic power provided by a symbolic software package.

For this purpose, a Mathematica 4.1 [9] function is given below to illustrate the theoretical as well as computational properties of the method.

To study the non-numerical properties of the method, a static implementation (with a preset value of $n$ ) is given.

$$
\begin{aligned}
& \text { Romberg[f[ } \left.\left.x_{-}\right],\left\{\mathrm{x}_{-}, \mathrm{a}_{-}, \mathrm{b}_{-}\right\}, \mathrm{n}_{-}\right]:= \\
& \quad(\mathrm{h}=\mathrm{b}-\mathrm{a} ; \\
& \quad t[1]=\frac{1}{2} h(f[a]+f[b]) ; \\
& \quad \operatorname{romb}[1,1]=\mathrm{t}[1] ; \\
& \quad \operatorname{For}\left[m=2, m \leq n, m++, k=m-1 ; D o\left[x[j]=a+\frac{(j-i) h}{2^{k}},\left\{j, 1,2^{k}+1\right\}\right] ;\right.
\end{aligned}
$$




$$
\left.t[m]=\frac{1}{2} t[m-1]+\frac{h \sum_{j=1}^{2^{(k-1)}} f[x[2 j]]}{2^{k}}\right] ;
$$

For $[\mathrm{m}=2, \mathrm{~m} \leq \mathrm{n}, \mathrm{m}++$, romb $[\mathrm{m}, 1]=\mathrm{t}[\mathrm{m}]]$;

For $[i=2, i \leq n, i++$,

$$
\text { For } \left.\left.\left[j=2, j \leq i, j++\operatorname{romb}[i, j]=\frac{4^{j-1} \operatorname{romb}[i, j-1]-\operatorname{romb}[i-1, j-1]}{4^{j-1}-1}\right]\right] ;\right)
$$

The symbolic power of Mathematica now can be facilitated to study the method for a symbolic function $g$ over $[a, b]$ by calling Romberg for, say, $n=10$ levels of subdivision using Romber $g[g[x],\{x, a, b\}, 10]$.

\section{Experiments}

A sample Mathematica 4.1 session is arranged to demonstrate the properties of the Romberg integration for a general function $g$. The following input commands are to be executed following the call to Romberg function.

- Set up the first trapezoidal approximation $t[1]$ to I over [a,b].

$\operatorname{In}[1]:=\mathrm{t}[1]$

$\frac{1}{2}(-a+b)(g[a]+g[b])$

- Display the second approximation $t[2]$ over 2 sub-intervals

In [2] :=Simplify [t [2] ]

$-\frac{1}{4}(a-b)\left(g[a]+g[b]+2 g\left[\frac{a+b}{2}\right]\right)$

- Set up the composite trapezoidal rule $t[3]$ over 4 sub-intervals.

$\operatorname{In}[3]:=\mathrm{t}[3]$

$\frac{1}{2}\left(\frac{1}{4}(-a+b)(g[a]+g[b])+\frac{1}{2}(-a+b) g\left[a+\frac{1}{2}(-a+b)\right]\right)+$

$\frac{1}{4}(-a+b)\left(g\left[a+\frac{1}{4}(-a+b)\right]+g\left[a+\frac{3}{4}(-a+b)\right]\right)$

- Simplify the expression

In [4]:=Simplify [\%]

$-\frac{1}{8}(a-b)\left(g[a]+g[b]+2\left(g\left[\frac{a+b}{2}\right]+g\left[\frac{1}{4}(3 a+b)\right]+g\left[\frac{1}{4}(a+3 b)\right]\right)\right)$

- Display a first level extrapolation (Simpson's rule over $[\mathrm{a}, \mathrm{b}]$ ).

In [5] :=Simplify [4t [2] -t [1] ) /3]

$-\frac{1}{6}(a-b)\left(g[a]+g[b]+4 g\left[\frac{a+b}{2}\right]\right)$

- Compare Out[5] with the value of romb[2,2].

In [6] : =Simplify $[\operatorname{romb}[2,2]]$

$-\frac{1}{6}(a-b)\left(g[a]+g[b]+4 g\left[\frac{a+b}{2}\right]\right)$

- Display the value of romb[3,2] (Simpson's rule applied to 2 sub-intervals)

In [7] :=Simplify [romb $[3,2]]$

$-\frac{1}{12}(a-b)\left(g[a]+g[b]+2 g\left[\frac{a+b}{2}\right] 4 g\left[\frac{1}{4}(3 a+b)\right]+4 g\left[\frac{1}{4}(a+3 b)\right]\right)$ 
- Display the value of romb[3,3] (first entry in the third column of the Romberg table). Observe that this is Newton's interpolatory formula.

In [8] :=Simplify [romb $[3,3]]$

$-\frac{1}{90}(a-b)\left(7 g[a]+7 g[b]+4\left(3 g\left[\frac{a+b}{2}\right]+8 g\left[\frac{1}{4}(3 a+b)\right]+8 g\left[\frac{1}{4}(a+3 b)\right]\right)\right)$

- Compute the integral below by displaying romb[6,6]. Estimate the err(or) by comparing the result with that of Mathematica's Integrate (actual).

$$
\int_{0}^{\frac{\pi}{2}} \cos x d x=2
$$

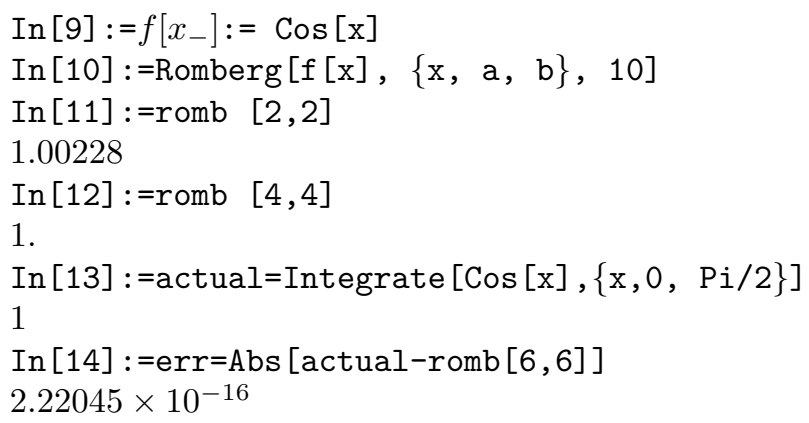

- Trapezoidal rule is linear and, therefore, integrates linear polynomials exactly, and each Romberg column doubles the order. To investigate this let $f$ be $x^{\wedge} 7$, over $[0,1 / 2]$, and observe that $\operatorname{romb}[4, j]$ is exact $(1 / 2048=$ $0.000488281)$.

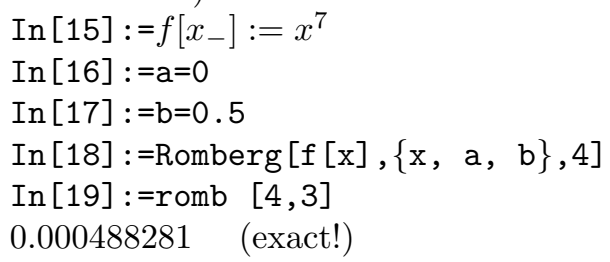

\section{Justification of the Method}

Romberg extrapolation method is based upon the existence of the asymptotic error expansion discussed in Section 2. Assuming the existence of such an error expansion, Mathematica's Sum, and Expansion functions can be used to illustrate how the method works by symbolically deriving expressions for the entries of the Romberg table. The commands written for this purpose are given below:

$\operatorname{In}[20]:=$ Array $[c, 4]$

$\{c[1], c[2], c[3], c[4]\}$

$\operatorname{In}[21]:=e\left[h_{-}\right]:=\operatorname{Sum}\left[c[i] h^{\wedge}(2 i),\{i, 1,4\}\right]$

$\operatorname{In}[22]:=p=(4 e[h / 2]-e[h]) / 3$ 
$\frac{1}{3}\left(-h^{2} c[1]-h^{4} c[2]-h^{6} c[3]-h^{8} c[4]+4\left(\frac{h^{2} c[1]}{4}+\frac{h^{4} c[2]}{16}+\frac{h^{6} c[3]}{64}+\frac{h^{8} c[4]}{256}\right)\right)$

$\operatorname{In}[23]:=\mathrm{q}=(4 \mathrm{e}[\mathrm{h} / 4]-\mathrm{e}[\mathrm{h} / 2]) / 3$

$\frac{1}{3}\left(-\frac{h^{2} c[1]}{4}-\frac{h^{4} c[2]}{16}-\frac{h^{6} c[3]}{64}-\frac{h^{8} c[4]}{256}+4\left(\frac{h^{2} c[1]}{16}+\frac{h^{4} c[2]}{256}+\frac{h^{6} c[3]}{4096}+\frac{h^{8} c[4]}{65536}\right)\right)$

In $[24]:=$ Expand [Simplify $[\mathrm{p}]]$

$-\frac{1}{4} h^{4} c[2]-\frac{5}{16} h^{6} c[3]-\frac{21}{64} h^{8} c[4]$

In [25] : =Expand [Simplify [16q-p)/15]]

$\frac{1}{64} h^{6} c[3]+\frac{21 h^{8} c[4]}{1024}$

The last two results show that the accuracy of the values in the second and third columns of the Romberg table are of $O\left(h^{4}\right)$, and $O\left(h^{6}\right)$, respectively.

\section{About the Error Term}

Mathematica function Series can be used to verify the error term of the basic Trapezoidal rule given by

$$
E=-\frac{h^{2}}{12}\left[f^{\prime}(b)-f^{\prime}(a)\right]+\frac{(b-a) h^{4}}{720} f^{(4)}(\mu), \quad \mu \epsilon[a, b]
$$

In order to verify the error, consider $S=\int_{a}^{a+h} \operatorname{Sin}[x] d x=$ $-\operatorname{Cos}[a+h]+\operatorname{Cos}[a]$ and perform the following input commands:

$\operatorname{In}[26]:=f\left[x_{-}\right]:=\operatorname{Sin}[x]$

In [27] : =Romberg[f[x], $\{\mathrm{x}, \mathrm{a}, \mathrm{a}+\mathrm{h}\}, 6]$

$\operatorname{In}[28]:=\mathrm{t}[1]$

$\frac{1}{2} h(\operatorname{Sin}[a]+\operatorname{Sin}[a+h])$

In [29] : $=\mathrm{s}=$ Integrate[Sin $[\mathrm{x}],\{\mathrm{x}, \mathrm{a}, \mathrm{a}+\mathrm{h}\}]$

$\operatorname{Cos}[\mathrm{a}]-\operatorname{Cos}[\mathrm{a}+\mathrm{h}]$

$\operatorname{In}[30]:=\mathrm{e}=$ Series[s-t[1], $\{\mathrm{h}, 0,3\}]$

$\frac{1}{12} \operatorname{Sin}[a] h^{3}+O[h]^{4}$

In [31] : =terror $=-\mathrm{h}^{\wedge} 2 / 12(\operatorname{Cos}[\mathrm{a}+\mathrm{h}]-\operatorname{Cos}[\mathrm{a}])$

$-\frac{1}{12} h^{2}(-\operatorname{Cos}[a]+\operatorname{Cos}[a+h])$

$\operatorname{In}[32]:=$ Series [terror, $\{\mathrm{h}, 0,3\}$ ]

$\frac{1}{12} \operatorname{Sin}[a] h^{3}+O[h]^{4}$

Observe that error expression above is identical with the dominant term of the error formula (7).

\section{Computational Complexity of Romberg Integration}

The complexity of any numerical integration algorithm is mainly depicted by the number of function evaluations at the nodes of integration. In Romberg integration, from level i-1 to i, $2^{i}$ additional integrand evaluations are required. In higher dimensions, this causes too many function evaluations, and the method 
becomes computationally inefficient. A dynamic implementation minimizes the cost of the algorithm. That is, at each level, rows of the table are completed by the Romberg formula and an error test is performed to check the accuracy of the diagonal value $\operatorname{rom} b[i, i]$. Once the error criteria is satisfied, the algorithm terminates avoiding further subdivisions and function evaluations. The dynamic implementation is given below:

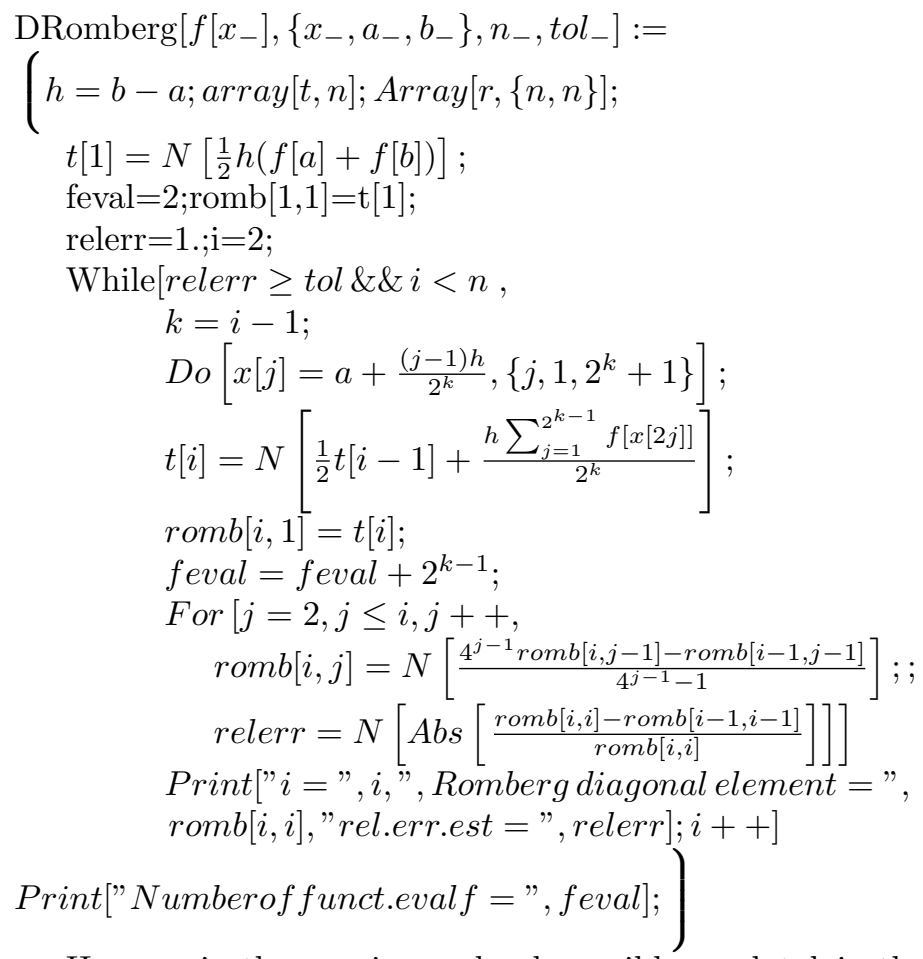

Here, $\mathrm{n}$ is the maximum level possible, and tol is the user-defined error tolerance. A sample run and its output are given for the integration of $e^{\cos x}$, over $[0,2]$ with the error tolerance value $=0.00001$ (exact value $=3.454359)$.

$\operatorname{In}[1]:=f\left[x_{-}\right]:=\operatorname{Exp}[\operatorname{Cos}[x]]$

In [2] :=DRomberg $[\mathrm{f}[\mathrm{x}],\{\mathrm{x}, 0,2\}, 10,0.00001]$

$\mathrm{i}=2$, Romberg diagonal element $=3.41466$, rel. err. est. $=0.0107744$

$\mathrm{i}=3$, Romberg diagonal element $=3.4567$, rel. err. est. $=0.0121627$

$\mathrm{i}=4$, Romberg diagonal element $=3.45432$, rel. err. est. $=0.000688374$

$\mathrm{i}=5$, Romberg diagonal element $=3.45436$, rel. err. est. $=9.83452 \times 10^{-6}$

Number of funct. eval $f=17$

\section{Cautious Extrapolation}

The basic assumption in the preceding discussion is the existence of the error expansion given by (5). If such an expansion is not valid (for end point singu- 
larities, and singularities in the derivatives of f) then Romberg fails to provide the required accuracy or diverges. Singularities can be detected dynamically by providing a control over the values of the Romberg table. This process is known as cautious extrapolation [10] and described briefly in the sequel. Define,

$$
d[i, j]=\operatorname{romb}[i, j]-\operatorname{romb}[i-1, j], \quad i=2,3, \ldots \quad \text { and } \quad j=1,2, \ldots, i
$$

Assuming that the error expansion given by (5) holds, one obtains romb $[i, j]$ values satisfying the equations below:

$$
I-\operatorname{romb}[i, j]=C_{j+1}\left(\frac{h}{2^{i}}\right)^{2(j+1)}+O\left(h^{2(j+2)}\right)
$$

where $C_{j}$ 's are constants (i.e. independent of $h$ ). Using (8) and (9) and after some algebraic manipulations one obtains

$$
\begin{aligned}
d[i, j-1] & =C_{j}\left(\frac{h}{2^{i}}\right)^{2 j}-C_{j}\left(\frac{h}{2^{i-1}}\right)^{2 j}+O\left(h^{2(j+1)}\right) \\
& =C_{j} h^{2 j}\left(1-4^{j}\right) \cdot 4^{-i j}+O\left(h^{2(j+1)}\right)
\end{aligned}
$$

and

$$
d[i-1, j-1]=C_{j} h^{2 j}\left(1-4^{j}\right) \cdot 4^{-(i-1) j}+O\left(h^{2(j+1)}\right)
$$

Therefore, as $\mathrm{j}$ (depth of extrapolation) increases, one gets

$$
\frac{d[i-1, j-1]}{d[i, j-1]} \rightarrow \frac{4^{-(i-1) j}}{4^{-j i}}=4^{j}
$$

Consequently, before computing a new Romberg column, one should first check whether the ratio above approximates $4^{j}$ or not. A typical test condition can be specified as

$$
|| \frac{d[i-1, j-1]}{d[i, j-1]}\left|-4^{j}\right| \leq t o l \cdot 4^{j}
$$

where $t o l$ is the user specified tolerance value, say 0.1 for one digit accuracy, in the ratio.

Again, the symbolic power of Mathematica is used to verify the cautious extrapolation. Consider the following Mathematica call to display one of the first column differences $(i=1)$ for a symbolic function $f\left[x_{-}\right]:=g[x]$.

$\operatorname{In}[3]:=\operatorname{Print}[\operatorname{Simplify}[(\operatorname{romb}[2,1]-\operatorname{romb}[1,1]) /(\operatorname{romb}[3,1]-\operatorname{romb}[2,1])]]$ $\frac{2\left(g[a]+g[b]-2 g\left[\frac{a+b}{2}\right]\right)}{g[a]+g[b]+2 g\left[\frac{a+b}{2}\right]-2 g\left[\frac{1}{4}(3 a+b)\right]-2 g\left[\frac{1}{4}(a+3 b)\right]}$ 
Using a series expansion around (a-b) and taking into account the first 2 terms, one obtains

$\operatorname{In}[4]:=$ Simplify $y\left[\right.$ Series $\left.\left[\frac{2\left(g[a]+g[b]-2 g\left[\frac{a+b}{2}\right]\right)}{g[a]+g[b]+2 g\left[\frac{a+b}{2}\right]-2 g\left[\frac{1}{4}(3 a+b)\right]-2 g\left[\frac{1}{4}(a+3 b)\right]},\{a, b, 2\}\right]\right]$

$4-\frac{g^{(4)}[b](a-b)^{2}}{16 g^{\prime \prime}[b]}+O[a-b]^{3}$

Observe that the first term of the expansion is $4^{\wedge} 1$ as expected. For the second column differences $(i=2)$ the expected value of the ratio is $4^{\wedge} 2=16$ ignoring the other terms of expansion. This is illustrated below:

In $[5]:=$ Simplify $\left[\right.$ Series $\left.\left[\frac{\operatorname{romb}[3,2]-\operatorname{romb}[2,2]}{\operatorname{romb}[4,2]-\operatorname{romb}[3,2]},\{a, b, 2\}\right]\right]$

$16-\frac{3 g^{(6)}[b](a-b)^{2}}{8 g^{(4)}[b]}+O[a-b]^{3}$

To show the same numerically, the integrand $f\left[x_{-}\right]:=x^{\wedge} 4$ over $[0,1]$ is considered, and a call to Romberg is made. The first 2 column differences are computed to be 3.90551 and 16 respectively. The first difference is close to 4 , whereas the second difference is 16 as expected (since for this function fifth (and higher order) derivatives are zero).

The second test involves integration of a function with derivatives that vary greatly in magnitude, $f\left[x_{-}\right]:=1 / \sqrt{|x|}$ over $[-9,10000]$. The differences $(2.10058,2.13913$, and 2.14663$)$ are computed for the first three columns of the table respectively $(i=1,2$, and 3 ). Obviously, these values are considerably far from the expected ones because of the singularity. In this case, Romberg method should work cautiously to produce an accurate result since the first term in the error expansion formula is no longer $h^{2}$. Exact value of the integral is 206, and $\operatorname{romb}[10,10]=200.566$ displaying slow convergence.

Now, consider an integral where f has an end-point singularity.

$$
I=\int_{0}^{1} \frac{\cos x}{\sqrt{x}} d x
$$

Since $f(x)$ is not defined at $x=0$, the Romberg algorithm terminates with the following error message.

\section{Power::infy : Infinite expression 1/0 encountered.}

In order to estimate this integral accurately, one alternative would be to pick a small positive number, a, and compute the following integral to obtain an approximate value

$$
I=\int_{a}^{1} \frac{\cos x}{\sqrt{x}} d x
$$

Using the Romberg function with $\mathrm{a}=0.000001$ one gets romb $[4,4]=39.6507$ as the integral value whereas the real value of the integral is 1.809048476 . Although 
the integral is continuous and bounded in the interval [0.000001,1], the difficulty arises from large variations in values of the integrand near the lower end of the integral. This erratic behavior is due to large variations in derivatives. One way to remedy this deficiency is to introduce more subdivisions of the interval. For example, for the same integral, values of romb[11,11] and romb[16,16] are 2.06852 and 1.81138, respectively providing better approximations to the integral.

Each singularity requires a special attention. In this particular case, one may use the substitution, $t=x^{0.5}$ to transform the integral to a nicely behaving one.

$$
I=\int_{0}^{1} 2 \cos t^{2} d t
$$

\section{Conclusions}

In this article, Romberg integration method is considered as a case study in order to present a highly technical method in a simplified manner by facilitating Mathematica. The concepts and the methodology adopted here can be easily applied to numerical differentiation, interpolation, and approximation among the many others.

Although the presentation does not involve any rigorous proofs at all, some hints are automatically provided by the symbolic derivations and justifications.

For the treatment of singularities, cautious extrapolation is also discussed and illustrated symbolically and numerically. As an ongoing research project the authors are investigating singular integrals using Mathematica. The results from this project will be reported elsewhere.

\section{References}

1. Joyce, D.C.: Survey of Extrapolation Processes in Numerical Analysis, SIAM Review, 13, 4 (1971) 435-490.

2. Romberg. W.: Vereinfachte Numerische Integration, Kgl. Nordske Vid. Selsk. Forh, bf 28 (1955) 30-36.

3. Yazıcı, A.: On the Subdivision Sequences of Extrapolation Method of Quadrature, METU Journal of Pure and Applied Sciences, 23, 1 (1990) 35-51.

4. Burden, R.L. and Faires, J.D.: Numerical Analysis, 3rd. Ed., PWS Publishers (1985).

5. Kelch, R.: Numerical Quadrature by Extrapolation with Automatic Result Verification, in Scientific Computing with Automatic result Verification, Academic Press, Inc. (1993) 143-185.

6. Lyness, J.N. and Mc Hugh, B.J.J.: On the Remainder Term in the N-Dimensional Euler-Maclaurin Expansion, Num.Math., 15 (1970) 333-344.

7. Skeel, R.D. and Keiper, J.B.: Elementary Numerical Computing with Mathematica, McGraw-Hill (1993).

8. Mathews, J.H. and Fink, K.D.: Numerical Methods Using Matlab, 3rd Edition, Prentice Hall (1999).

9. Wolfram, S.: The Mathematica Book, Cambridge University Press (1999).

10. Johnston, R.L.: Numerical Methods: A Software Approach, John Wiley and Sons (1982). 